PROCEEDINGS OF THE

AMERICAN MATHEMATICAL SOCIETY

Volume 131, Number 8, Pages 2415-2422

S 0002-9939(02)06867-3

Article electronically published on November 27, 2002

\title{
SOME REMARKS RELATED TO DE GIORGI'S CONJECTURE
}

\author{
YIHONG DU AND LI MA
}

(Communicated by David S. Tartakoff)

\begin{abstract}
For several classes of functions including the special case $f(u)=$ $u-u^{3}$, we obtain boundedness and symmetry results for solutions of the problem $-\Delta u=f(u)$ defined on $R^{n}$. Our results complement a number of recent results related to a conjecture of De Giorgi.
\end{abstract}

\section{INTRODUCTION}

In this paper, we make several observations related to the following conjecture of De Giorgi $\mathrm{dG}$ ]: If $u$ is a solution of the scalar Ginzburg-Landau equation

$$
\Delta u+u\left(1-u^{2}\right)=0 \text { on } \quad R^{n}
$$

such that $|u| \leq 1$ and $\partial_{n} u>0$ on $R^{n}$, and

$$
\lim _{x_{n} \rightarrow \pm \infty} u\left(x^{\prime}, x_{n}\right)= \pm 1, \forall x^{\prime} \in R^{n-1},
$$

then all level sets of $u$ are hyperplanes, at least for $n \leq 8$. Here $\partial_{n} u$ denotes the partial derivative of $u$ with respect to $x_{n}$, the last component of $x$, and $x^{\prime}$ denotes the first $n-1$ components of $x$.

When $n=2$, this conjecture was completely resolved by Ghoussoub and Gui GG]. When $n=3$, it was very recently proved by Ambrosio and Cabre [AC]. Both solutions of the conjecture are based on a Liouville-type theorem due to Berestycki, Caffarelli and Nirenberg [BCN2]. The first partial answer to the De Giorgi conjecture is from the work in 1980 of Modica and Mortola [MM]. In 1985, Modica found a pointwise gradient bound for all bounded solutions. This estimate was further generalized by Caffarelli, Garofalo and Segala [CGS] to more general nonlinear partial differential equations which include the p-Laplacian. Under more assumptions on the solutions, for example, if $u(x)=u\left(x^{\prime}, x_{n}\right) \rightarrow \pm 1$ as $x_{n} \rightarrow \pm \infty$ holds uniformly for $x^{\prime} \in R^{n-1}$, the conclusion of this conjecture was confirmed in $[\overline{\mathrm{BBG}}$, $[\overline{\mathrm{BHM}}]$ and $[\mathrm{F} 1$ for any $n \geq 2$. The conjecture in its original form, however, remains open for $n>3$. We refer to [AAC] for a fuller account of the history and progress about this conjecture.

Received by the editors March 10, 2002.

2000 Mathematics Subject Classification. Primary 35J15, 35J60.

Key words and phrases. Elliptic equation, maximum principle, symmetry of solution.

The first author was partially supported by the Australian Academy of Science and Academia Sinica under an exchange program while part of this work was carried out. The second author was partially supported by a grant from the national 973 project of China and a scientific grant of Tsinghua University at Beijing. 
All these previous studies obtain results which are valid not only for the special nonlinearity appearing in (1.1), but also for much more general nonlinearities. In this paper, we show that for some narrower classes of nonlinearities containing the one in (1.1), several further results can be obtained.

In order to describe our results, let us recall that for the special nonlinearity in (1.1), the condition $|u| \leq 1$ in De Giorgi's conjecture is unnecessary. In fact, the following result holds.

Theorem 1.1. Suppose $u \in C^{2}\left(R^{n}\right)$ is a solution of (1.1). Then $|u| \leq 1$. Moreover, $u$ is either a constant (hence, $u \equiv 1$, or $u \equiv 0$, or $u \equiv-1$ ), or $u$ changes sign and satisfies $|u|<1$ on $R^{n}$.

The first conclusion in Theorem 1.1 follows from Proposition 1.9 in [F2], and the rest is a consequence of Theorem 3.1 in [AW]. Our first result extends Theorem 1.1 to more general nonlinearities (see Theorem 2.1 below) though somewhat less general than those in $\mathrm{GG},[\mathrm{BBG}], \overline{\mathrm{BHM}}],[\mathrm{F} 1],[\mathrm{AC}]$. This is not obvious from the proof in [F2], but it follows from a simple application of boundary blow-up solutions, as in $[\mathrm{DM}]$.

Our second result is motivated by some recent results in $[\mathrm{BBG},[\mathrm{BHM}$ and F1. One of the main results in these papers says that if $u$ solves (1.1) and satisfies $|u| \leq 1$ and

$$
u\left(x^{\prime}, x_{n}\right) \rightarrow \pm 1 \text { as } x_{n} \rightarrow \pm \infty \text { uniformly in } x^{\prime} \in R^{n-1},
$$

then $u(x)=u\left(x_{n}\right)$. Farina [F1] further observes that, under the restrictions already put on the nonlinearity, a result in [BCN1] implies that (1.2) is equivalent to

$$
x_{n} u\left(x^{\prime}, x_{n}\right)>0, \forall\left|x_{n}\right|>R, \forall x^{\prime} \in R^{n-1},
$$

for some $R>0$. Or equivalently, the zero set of $u$, denoted by $u^{-1}(0)$, lies between two parallel hyperplanes $\left\{x_{n}=-R\right\}$ and $\left\{x_{n}=R\right\}$. This somehow relates the above result to Theorem 2 in $[\mathrm{BBG}$, which improves a result in [MM and asserts that if a solution $u$ to (1.1) satisfies $|u| \leq 1$ and $\partial_{n} u>0$ and that all its level sets are graphs of Lipschitzian functions of $x^{\prime}$, then $u$ is a function of one variable.

All these above-mentioned results are proved for rather general nonlinearities. Under some further restrictions, we have a result which implies the following.

Theorem 1.2. Suppose that $u \in C^{2}\left(R^{n}\right)$ solves (1.1) on $R^{n}$. If $u^{-1}(0)$ lies on one side of a hyperplane and touches that hyperplane, i.e., there exists $\nu \in S^{n-1}$ and $x_{0} \in u^{-1}(0)$ such that $\nu \cdot\left(x-x_{0}\right) \geq 0$ for all $x \in u^{-1}(0)$, then $u$ depends on one variable only (in the direction of $\nu$ ).

Note that, in Theorem 1.2, a condition of the type $\partial_{n} u>0$ is not required. Moreover, no regularity on $u^{-1}(0)$ is assumed. In our proof of Theorem 1.2, the oddness of the nonlinearity will be employed.

We end this Introduction with the statement of a simple consequence of Theorem 1.2. Assume that $M$ is the set of one-dimensional solutions of (1.1). Then $M$ can be explicitly expressed in the following way. Let $v=v(t)$ be the function $\tanh (t / \sqrt{2})$, which is, up to a translation, the unique solution of the problem

$$
h^{\prime \prime}=h^{3}-h, h( \pm \infty)= \pm 1 .
$$

For $a \in S^{n-1}$ and $c \in R$, we let

$$
u_{a, c}(x)=\tanh ((a \cdot x-c) / \sqrt{2}) .
$$


Then

$$
M=\left\{u_{a, c} ; a \in S^{n-1}, c \in R\right\} .
$$

Corollary 1.3. Assume that $u \in C^{2}\left(R^{n}\right)$ solves (1.1) and the $C^{0}$-distance of $u$ to $M$ is less than 1, i.e.,

$$
\inf _{v \in M} \sup _{x \in R^{n}}|u(x)-v(x)|<1
$$

Then $u \in M$.

Note that $M$ is a closed set in the $C^{0}$-topology, and if we denote the $C^{0}$ distance function by dist 0 , then, by Theorem 1.1, for any solution $u$ of $(1.1), \operatorname{dist}_{0}(u, M) \leq 2$. In particular, if $u=0$, then $\operatorname{dist}_{0}(u, M)=1$, and if $u=1$ or $u=-1$, then $\operatorname{dist}_{0}(u, M)=2$.

The rest of this paper is organized as follows. In section 2 , we present a result (Theorem 2.1) which improves Theorem 1.1. Theorem 1.2 will be discussed in section 3 , as a special case of a more general result.

\section{Global Boundedness and Related Results}

In this section, we prove a general result which contains Theorem 1.1 as a special case. We consider the problem

$$
-\Delta u=f(u), \quad x \in R^{n},
$$

where the real valued $C^{1}$ function $f$ is assumed to satisfy

$$
\left\{\begin{array}{l}
f(0)=f(1)=f(-1)=0, \\
u f(u)>0 \text { for } 0<|u|<1, u f(u)<0 \text { for }|u|>1,
\end{array}\right.
$$

and for some large constant $M>1$

$$
\left\{\begin{array}{l}
\underline{\lim }_{u \rightarrow 0} \frac{f(u)}{u|u|^{2 / n}} \in(0, \infty] \\
u f(u) \leq u g(|u|)<0 \text { for }|u|>M, \\
g(u) \text { is decreasing in }[M, \infty) \text { and } \int_{M}^{\infty}\left[\int_{M}^{u}|g(s)| d s\right]^{-1 / 2} d u<\infty
\end{array}\right.
$$

It is easily checked that if $p>0$, then $f(u)=u\left(1-|u|^{p}\right)$ satisfies both $(2.2)$ and (2.3).

Theorem 2.1. Suppose $f$ is $C^{1}$ and satisfies (2.2) and (2.3). Let $u \in C^{2}\left(R^{n}\right)$ be a solution of (2.1). Then the conclusions in Theorem 1.1 hold.

Proof. From Theorem 5.2 in $[\mathrm{DM}]$ and our assumptions on $f$ and the Harnack inequality, we know that any solution $u$ which does not change sign in $R^{n}$ must be constant, that is, $u \equiv 1$ or $u \equiv-1$ or $u \equiv 0$. Therefore we only need to consider solutions of (2.1) which changes sign in $R^{n}$.

Suppose now $u$ is a sign-changing solution of (2.1). We want to show that $|u|<1$ on $R^{n}$. Let us first observe that it suffices to show $|u| \leq 1$ in $R^{n}$. Indeed, if $\left|u\left(x_{0}\right)\right|=1$, say $u\left(x_{0}\right)=-1$, then $w:=u+1$ satisfies

$$
-\Delta w=c(x) w, w \geq 0, w\left(x_{0}\right)=0,
$$

where $c \in L^{\infty}\left(R^{n}\right)$ is given by $c(x)=[f(u(x))-f(-1)] /(u(x)+1)$ when $u(x) \neq-1$, and $c(x)=f^{\prime}(-1)$ otherwise. Hence it follows from the Harnack inequality that $w \equiv 0$, contradicting our assumption that $u$ changes sign.

To show $|u| \leq 1$ on $R^{n}$, we adapt the argument used in the proof of Theorem 5.2 in $\mathrm{DM}$. 
Let $h(u)=-g(u+M)$ for $u \geq 0$ and $h(u)=-g(M)$ for $u<0$. Then $h$ is positive and due to (2.3), we can use the proof of Theorem 1 of Keller [Ke] to conclude that the problem

$$
\Delta u=h(u),\left.u\right|_{\partial B}=\infty
$$

has a unique positive solution $u_{\infty}$, where $B$ stands for a ball centered at the origin with small radius (the proof in [Ke] actually shows that $\min _{R^{n}} u_{\infty} \rightarrow \infty$ as the radius of $B$ goes to 0$)$. It follows that $v_{\infty}=u_{\infty}+M$ is a positive solution to

$$
-\Delta v=g(v),\left.v\right|_{\partial B}=\infty \text {. }
$$

We claim that $u \leq c:=\min _{B} v_{\infty}(x)$ on $R^{n}$. Otherwise, we can find $x_{0} \in R^{n}$ such that $u\left(x_{0}\right)>c$. Define $v(x)=v_{\infty}\left(x-x_{0}\right)$. We find that the set $\{x \in$ $\left.B\left(x_{0}\right): u(x)>v(x)\right\}$ has a component $\Omega$ whose closure lies entirely in the open ball $B\left(x_{0}\right)=\left\{x: x-x_{0} \in B\right\}$. On $\Omega$, we have $u(x)>v(x) \geq c>M$ and $\Delta u+g(u) \geq 0=\Delta v+g(v)$. Moreover, $u=v$ on $\partial \Omega$. As $g(u)$ is decreasing for $u>M$, from

$$
\Delta(u-v)+c(x)(u-v) \geq 0, c(x)=[g(u)-g(v)] /(u-v) \leq 0 \text { on } \Omega
$$

and the maximum principle, we deduce that $u \equiv v$ in $\Omega$. This contradiction shows that we must have $u \leq c$ on $R^{n}$.

Applying the above argument to $w=-u$ which satisfies

$$
-\Delta w=g(w), g(w)=-f(-w),
$$

we deduce that $u \geq-c$ on $R^{n}$. Therefore we have

$$
-c \leq u(x) \leq c, \forall x \in R^{n} .
$$

Let $u_{c}$ and $u_{-c}$ denote the unique solution of

$$
u^{\prime}=f(u), u(0)=u_{0},
$$

with $u_{0}=c$ and $u_{0}=-c$, respectively. Then it follows from elementary analysis that $u_{c}(t) \rightarrow 1$ and $u_{-c}(t) \rightarrow-1$ as $t \rightarrow+\infty$. On the other hand, $u, u_{c}$ and $u_{-c}$ are all bounded solutions of the parabolic problem

$$
u_{t}-\Delta u=f(u) \text {. }
$$

Since $u_{c}(0) \geq u(x) \geq u_{-c}(0)$ on $R^{n}$, by the parabolic maximum principle and the boundedness of $u, u_{c}$ and $u_{-c}$ ([Fr Theorem 9, page 43]), we conclude that $u_{-c}(t) \leq u(x) \leq u_{c}(t)$ for all $t>0$. Letting $t \rightarrow \infty$, we obtain $-1 \leq u(x) \leq 1$, as required. This finishes our proof of Theorem 2.1.

Remark 2.2. As was remarked in [DM], the behavior of the function $f$ near infinity described by (2.3) is necessary for Theorem 2.1 to hold true. One can find functions $f$ which satisfy all the other conditions in (2.2) and (2.3) except the restriction near infinity in (2.3), such that (2.1) has unbounded entire positive and negative solutions. The interested reader may check $[\mathrm{DM}]$ for more details.

Remark 2.3. By using the recent result in [DD] instead of [AW] (see page 121 in DM]), we can show that the restriction

$$
\varliminf_{u \rightarrow 0} \frac{f(u)}{u|u|^{2 / n}} \in(0, \infty]
$$


in condition (2.3) can be relaxed to

$$
\varliminf_{u \rightarrow 0} \frac{f(u)}{u|u|^{2 /(n-2)}} \in(0, \infty]
$$

which is optimal.

\section{ODD NONLINEARITY AND RELATED RESUlTS}

In this section, we prove a generalization of Theorem 1.2.

We first recall two lemmas. The first one is from $\mathrm{BHM}$ which is a simple consequence of Lemma 2.1 in BCN1.

Lemma 3.1. Let $f$ be a Lipschitz continuous function which is non-increasing on $[-1,-1+\delta]$ and on $[1-\delta, 1]$ for some $\delta>0$. Assume that $u_{1}, u_{2}$ are solutions of (2.1) over some open connected set $\Omega \subset R^{n}$ and $\left|u_{i}\right| \leq 1(i=1,2)$. Assume further that $u_{2} \geq u_{1}$ on $\partial \Omega$ and either $u_{2} \geq 1-\delta$ or $u_{1} \leq-1+\delta$ in $\Omega$. Then $u_{2} \geq u_{1}$ in $\Omega$ provided that $R^{n} \backslash \Omega$ contains an infinite open connected cone.

The second is Lemma 2.4 in [F1] which follows from Lemmas 3.2 and 3.3 in BCN1].

Lemma 3.2. Let $f$ be a Lipschitz continuous function which is positive over $(0,1)$, and satisfies $f(1)=0, f(t) \geq \delta_{0} t$ on $\left(0, t_{0}\right]$ for some small $\delta_{0}>0$ and $t_{0}>0$. If $u$ is $C^{2}$ on the half plane $\Sigma_{M}:=\left\{x \in R^{n}: x_{n}>M\right\}$ and satisfies

$$
\Delta u+f(u) \leq 0,0<u \leq 1 \text { on } \Sigma_{M},
$$

then $u\left(x^{\prime}, x_{n}\right) \rightarrow 1$ uniformly in $x^{\prime} \in R^{n-1}$ as $x_{n} \rightarrow+\infty$.

Theorem 3.3. Suppose $f$ is Lipschitz continuous and satisfies

$$
f(-1)=f(0)=f(1)=0, \text { tf }(t)>0 \text { when } 0<|t|<1,
$$

and for some small positive constants $\delta_{0}, t_{0}$ and $\delta$,

$$
f(t) / t \geq \delta_{0} \text { when } 0<|t|<t_{0},
$$

$f$ is non-increasing on $[-1,-1+\delta] \cup[1-\delta, 1]$.

Furthermore, assume that $f(t)$ is odd in $t$. Then the statement in Theorem 1.2 holds for any solution $u \in C^{2}\left(R^{n}\right)$ of (2.1) satisfying $|u| \leq 1$.

Proof. Through a rotation and translation, we may assume that the hyperplane is given by $x_{n}=0, u(0)=0$ and $u^{-1}(0) \subset\left\{x: x_{n} \leq 0\right\}$. We may assume that $u\left(x^{\prime}, x_{n}\right)>0, \forall x^{\prime} \in R^{n-1}, \forall x_{n}>0$; the other possibility that $u\left(x^{\prime}, x_{n}\right)<0$, $\forall x^{\prime} \in R^{n-1}, \forall x_{n}>0$ can be handled analogously.

For $\tau \geq 0$, let us define

$$
u_{\tau}\left(x^{\prime}, x_{n}\right)=-u\left(x^{\prime}, 2 \tau-x_{n}\right) .
$$

Since $f$ is odd, we easily see that

$$
-\Delta u_{\tau}=f\left(u_{\tau}\right)
$$

Clearly

$$
\left.u\right|_{\left\{x_{n}=\tau\right\}} \geq 0 \geq\left. u_{\tau}\right|_{\left\{x_{n}=\tau\right\}} .
$$

We want to show that for every $\tau \geq 0, u \geq u_{\tau}$ on the half space $\left\{x: x_{n} \geq \tau\right\}$. 
Since $u(x)>0$ when $x_{n}>0$, it follows from Lemma 3.2 that $u\left(x^{\prime}, x_{n}\right) \rightarrow 1$ as $x_{n} \rightarrow+\infty$ uniformly in $x^{\prime} \in R^{n-1}$. Therefore, for large $\tau$ we can apply Lemma 3.1 to

$$
\Omega:=\left\{x: x_{n}>\tau\right\}
$$

to conclude that $u \geq u_{\tau}$ on $\Omega$. Now define

$$
\tau_{0}=\inf \left\{\tau \in[0, \infty): u\left(x^{\prime}, x_{n}\right) \geq u_{\tau}\left(x^{\prime}, x_{n}\right), \forall x^{\prime} \in R^{n-1}, \forall x_{n} \geq \tau\right\} .
$$

Claim: $\tau_{0}=0$.

Otherwise, $\tau_{0}>0$ and $u(x) \geq u_{\tau_{0}}(x)$ on the set $\Omega_{0}:=\left\{x: x_{n} \geq \tau_{0}\right\}$. Clearly $w:=u-u_{\tau_{0}}$ satisfies

$$
-\Delta w=c(x) w, w \geq 0, \forall x \in \Omega_{0},
$$

where $c \in L^{\infty}\left(\Omega_{0}\right)$. Since $u>0>u_{\tau_{0}}$ on $\partial \Omega_{0}$, by the definition of $\tau_{0}$, we have two possibilities:

(a) $w\left(x_{0}\right)=0$ for some $x_{0} \in \Omega_{0}$, or

(b) $w(x)>0$ in $\Omega_{0}$ and $w\left(z_{k}\right) \rightarrow 0$ for some $z_{k} \in \bar{\Omega}_{0}$ with $\left|z_{k}\right| \rightarrow \infty$.

If case (a) occurs, then the Harnack inequality forces $w \equiv 0$ on $\Omega_{0}$, which is impossible as $w>0$ on $\partial \Omega_{0}$.

If (b) occurs, we set $u_{k}(x)=u\left(x+z_{k}\right)$. By standard elliptic estimates, up to extraction of a subsequence, $u_{k}$ converges in $C_{l o c}^{2}\left(R^{n}\right)$ to a solution $u^{*}$ of $(2.1)$ as $k \rightarrow \infty$. Moreover,

$$
v:=u^{*}-u_{\tau_{0}}^{*}
$$

satisfies $v(0)=0$ and

$$
-\Delta v=c^{*}(x) v, v \geq 0, \forall x \in \Omega^{*},
$$

where $c^{*} \in L^{\infty}\left(\Omega^{*}\right)$ and $\Omega^{*}=\left\{x: x_{n}>\tau^{*}\right\}$ with $\tau^{*} \in[-\infty, 0]$ determined by (passing to a subsequence when necessary)

$$
\tau^{*}=-\lim _{k \rightarrow \infty} d\left(z_{k}, \partial \Omega_{0}\right) .
$$

If $0 \in \Omega^{*}$, then we obtain from the Harnack inequality that $v \equiv 0$ on $\Omega^{*}$, i.e.,

$$
u^{*}\left(x^{\prime}, x_{n}\right)=-u^{*}\left(x^{\prime}, 2 \tau_{0}-x_{n}\right), \forall x^{\prime} \in R^{n-1}, \forall x_{n}>\tau^{*} .
$$

Taking $x_{n}=\tau_{0}$ we deduce $u^{*}\left(x^{\prime}, \tau_{0}\right)=0$. This implies that $\left\{d\left(z_{k}, \partial \Omega_{0}\right)\right\}$ is bounded, for otherwise, due to $u\left(x^{\prime}, x_{n}\right) \rightarrow 1$ uniformly in $x^{\prime} \in R^{n-1}$ as $x_{n} \rightarrow+\infty$, we would have $u^{*} \equiv 1$. The boundedness of $\left\{d\left(z_{k}, \partial \Omega_{0}\right)\right\}$ and the fact that $u\left(x^{\prime}, x_{n}\right) \rightarrow 1$ uniformly in $x^{\prime} \in R^{n-1}$ as $x_{n} \rightarrow+\infty$ imply $u^{*}\left(x^{\prime}, x_{n}\right) \rightarrow 1$ uniformly in $x^{\prime} \in R^{n-1}$ as $x_{n} \rightarrow+\infty$. This together with (3.1) implies that $u^{*}\left(x^{\prime}, x_{n}\right) \rightarrow-1$ uniformly in $x^{\prime}$ as $x_{n} \rightarrow-\infty$. Hence we can use Theorem 1 in $\mathrm{BHM}$ to conclude that $u^{*}(x)=u^{*}\left(x_{n}\right)$ and is increasing in $x_{n}$. On the other hand, since $u_{k}(0)=u\left(z_{k}\right)>0$, we have $u^{*}(0) \geq 0$, a contradiction to the monotonicity of $u^{*}\left(x_{n}\right)$ and $u^{*}\left(\tau_{0}\right)=0$.

If $0 \in \partial \Omega^{*}$, we necessarily have $d\left(z_{k}, \partial \Omega_{0}\right) \rightarrow 0$ and hence $\tau^{*}=0, \Omega^{*}=\{x$ : $\left.x_{n}>0\right\}$. As before, this implies that $u^{*}\left(x^{\prime}, x_{n}\right) \rightarrow 1$ uniformly in $x^{\prime}$ as $x_{n} \rightarrow+\infty$. Moreover, for any $\eta \geq-\tau_{0}$, since $u_{k}\left(x^{\prime}, \eta\right)=u\left(\left(x^{\prime}, \eta\right)+z_{k}\right) \geq 0$, we deduce

$$
u^{*}\left(x^{\prime}, \eta\right) \geq 0, \forall x^{\prime} \in R^{n-1} .
$$

In particular,

$$
u^{*}\left(0, x_{n}\right) \geq 0, \forall x_{n} \geq-\tau_{0} .
$$


As $v(0)=0$, we have $u^{*}(0)=-u^{*}\left(0,2 \tau_{0}\right)$. Therefore we necessarily have $u^{*}(0)=$ $u^{*}\left(0,2 \tau_{0}\right)=0$. In view of $(3.2)$, the function $g(t):=u^{*}(0, t)$ has a local minimum at $t=0$ and at $t=2 \tau_{0}$. Therefore, $g^{\prime}(0)=g^{\prime}\left(2 \tau_{0}\right)=0$. This implies that $\partial_{n} v(0)=0$. Since $v$ satisfies

$$
-\Delta v=c^{*}(x) v, v \geq 0, \forall x \in \Omega^{*}, v(0)=0,0 \in \partial \Omega^{*},
$$

an application of the maximum principle and the Hopf boundary lemma gives $v \equiv 0$, i.e., $u^{*}\left(x^{\prime}, x_{n}\right)=-u^{*}\left(x^{\prime}, 2 \tau_{0}-x_{n}\right)$ for all $x^{\prime} \in R^{n-1}$ and all $x_{n} \geq 0$. We can now argue as in the case that $0 \in \Omega^{*}$ to conclude that $u^{*}(x)=u^{*}\left(x_{n}\right)$ and is increasing in $x_{n}$. But this is in contradiction with our earlier observation that $u^{*}(0)=u^{*}\left(2 \tau_{0}\right)$. This proves our Claim.

From $\tau_{0}=0$ we obtain $u\left(x^{\prime}, x_{n}\right) \geq-u\left(x^{\prime},-x_{n}\right)$ for all $x^{\prime} \in R^{n-1}$ and $x_{n} \geq 0$. Hence $w(x):=u\left(x^{\prime}, x_{n}\right)+u\left(x^{\prime},-x_{n}\right)$ satisfies

$$
-\Delta w=c(x) w, w \geq 0 \text { on } \Omega:=\left\{x: x_{n}>0\right\}, w(0)=0,
$$

where $c \in L^{\infty}(\Omega)$. By Harnack's inequality and the Hopf boundary lemma, we have either $w \equiv 0$ or $\partial_{n} w(0)>0$. A direct calculation yields

$$
\partial_{n} w(0)=\partial_{n} u(0)-\partial_{n} u(0)=0 .
$$

Hence we must have $w \equiv 0$ on $\Omega$, i.e., $u\left(x^{\prime}, x_{n}\right)=-u\left(x^{\prime},-x_{n}\right)$ for all $x^{\prime} \in R^{n-1}$ and all $x_{n}>0$. Recall that we have $u\left(x^{\prime}, x_{n}\right) \rightarrow 1$ uniformly in $x^{\prime} \in R^{n-1}$ as $x_{n} \rightarrow+\infty$. The above identity gives $u\left(x^{\prime}, x_{n}\right) \rightarrow-1$ uniformly in $x^{\prime}$ as $x_{n} \rightarrow-\infty$. Therefore we can use Theorem 1 of [BHM] and conclude that the proof of Theorem 4.2 is complete.

Remark 3.4. Clearly, if $f$ also satisfies the conditions in Theorem 2.1, then $|u| \leq 1$ is satisfied.

\section{ACKNOWLEDGMENTS}

L. Ma thanks Professor E.N. Dancer for inviting him to visit Sydney University. We also thank Professor Junping Shi for a useful remark regarding an earlier version of this paper.

\section{REFERENCES}

[AAC] G. Alberti, L. Ambrosio and X. Cabre, On a long-standing conjecture of E. De Giorgi: old and recent results, to appear in Acta Applicandae Mathematicae.

[AC] L. Ambrosio and X. Cabre, Entire solutions of semi-linear elliptic equations in $R^{3}$ and a conjecture of De Giorgi, Journal of Amer. Math. Soc., 13(2000), 725-739. MR 2001g:35064

[AW] D.G. Aronson and H.F. Weinberger, Multidimensional nonlinear diffusion arising in population genetics, Adv. Math., 30(1978), 33-76. MR 80a:35013

[BBG] M.T. Barlow, R.F. Bass and C. Gui, The Liouville property and a conjecture of De Giorgi, Comm. Pure Appl. Math., 53(2000), 1007-1038. MR 2001m:35095

[BCN1] H. Berestycki, L. Caffarelli and L. Nirenberg, Monotonicity for elliptic equations in an unbounded Lipschitz domain, Comm. Pure Appl. Math., 50(1997), 1089-1111. MR 98k:35064

[BCN2] H. Berestycki, L. Caffarelli and L. Nirenberg, Further properties for elliptic equations in unbounded domains, Ann. Scuola Norm. Sup. Pisa Cl. Sci. (4)25(1997), 69-94. MR 2000e:35053

[BHM] H. Berestycki, F. Hamel, and R. Monneau, One-dimensional symmetry of bounded entire solutions of some elliptic equations, Duke Math. J, 103(2000), 375-396. MR 2001j:35069 
[CGS] L. Caffarelli, N. Garofalo, and F. Segala, A gradient bound for entire solutions of quasilinear equations and its consequences, Comm. Pure Appl. Math., 47(1994), 1457-1473. MR 95k:35030

[DD] E.N. Dancer and Y. Du, Some remarks on Liouville type results for quasilinear elliptic equations, to appear in Proc. Amer. Math. Soc.

[dG] E. De Giorgi, Convergence problems for functionals and operators, Proc. Int. Meeting on Recent Methods in Nonlinear Analysis (Rome, 1978), E. de Giorgi et al. (eds.), Pitagora, Bologna (1979), pp. 131-188. MR 80k:49010

[DM] Y. Du and L. Ma, Logistic type equations on $R^{N}$ by a squeezing method involving boundary blow-up solutions, J. London Math. Soc., 64(2001), 107-124. MR 2002d:35089

[F1] A. Farina, Symmetry for solutions of semi-linear elliptic equations in $R^{N}$ and related conjectures, Rend. Mat. Acc. Lincei, 10(1999), 255-265. MR 2001g:35072

[F2] A. Farina, Finite-energy solutions, quantization effects and Liouville-type results for a variant of the Ginzburg-Landau systems in $R^{k}$, Diff. Integral Eqns., 11(1998), 975-893. MR 99j:35199

[Fr] A. Friedman, Partial Differential Equations of Parabolic Type, Prentice-Hall, New Jersey, 1964. MR 31:6062

[GG] N. Ghoussoub and C. Gui, On a conjecture of De Giorgi and some related problems, Math. Ann., 311(1998), 481-491. MR 99j:35049

[GT] D. Gilbarg and N.S. Trudinger, Elliptic Partial Differential Equations of Second Order, Springer-Verlag, Berlin/New York, 1983. MR 86c:35035, reprint MR 2001k:35004

[Ke] J.B. Keller, On solutions of $\Delta u=f(u)$, Comm. Pure Appl. Math., 10(1957), 503-510. MR 19:964c

[M] L. Modica, A gradient bound and a Liouville theorem for nonlinear Poisson equations, Comm. Pure Appl. Math., 38(1985), 679-684. MR 87m:35088

[MM] L. Modica and S. Mortola, Some entire solutions in the plane of nonlinear Poisson equations, Boll. Un. Mat. Ital., B, 17(1980), 614-622. MR 81k:35036

School of Mathematical and Computer Sciences, University of New England, ArmiDale, New South Wales 2351, Australia

E-mail address: ydu@turing.une.edu.au

Department of Mathematical Sciences, Tsinghua University, Beijing 100084, People's Republic of China

E-mail address: Ima@math.tsinghua.edu.cn 